\title{
Employing whole genome mapping for optimal de novo assembly of bacterial genomes
}

\author{
Basil Britto Xavier ${ }^{1}$, Julia Sabirova ${ }^{1}$, Moons Pieter ${ }^{1}$, Jean-Pierre Hernalsteens ${ }^{2}$, Henri de Greve ${ }^{3}$, \\ Herman Goossens ${ }^{1}$ and Surbhi Malhotra-Kumar ${ }^{1 *}$
}

\begin{abstract}
Background: De novo genome assembly can be challenging due to inherent properties of the reads, even when using current state-of-the-art assembly tools based on de Bruijn graphs. Often users are not bio-informaticians and, in a black box approach, utilise assembly parameters such as contig length and N50 to generate whole genome sequences, potentially resulting in mis-assemblies.

Findings: Utilising several assembly tools based on de Bruijn graphs like Velvet, SPAdes and IDBA, we demonstrate that at the optimal N50, mis-assemblies do occur, even when using the multi-k-mer approaches of SPAdes and IDBA. We demonstrate that whole genome mapping can be used to identify these mis-assemblies and can guide the selection of the best $k$-mer size which yields the highest N50 without mis-assemblies.

Conclusions: We demonstrate the utility of whole genome mapping (WGM) as a tool to identify mis-assemblies and to guide $k$-mer selection and higher quality de novo genome assembly of bacterial genomes.
\end{abstract}

Keywords: De-novo assembly, Whole genome mapping, k-mer, Microbial genomes, de bruijn graph, SPAdes and Velvet

\section{Findings}

Genome assembly is often a primary step in the process of yielding results that lead to interpretation of biological data and hence sub-optimally assembled genomes might lead to faulty conclusions [1]. Factors causing such low quality genome assembly include sequence quality, presence of repetitive sequences, base composition, size and low genome coverage [2,3], all of which complicate downstream data analysis using the available tools [4]. Currently, de novo assemblers based on de Bruijn graph are considered to yield the best results provided sufficient sequence quality and coverage are achieved. Such assembly tools based on de Bruijn graph algorithms, like Velvet [5] and SPAdes [6] use $k$-mers as building blocks, but as most users are not bio-informaticians, these tools are often considered as an encrypted black box with the quality of the assembly usually determined by statistics parameters such as the N50 and the size and number of contigs or scaffolds produced by the

\footnotetext{
* Correspondence: surbhi.malhotra@uantwerpen.be

${ }^{1}$ Department of Medical Microbiology, Vaccine \& Infectious Disease Institute, Universiteit Antwerpen, Antwerp, Belgium

Full list of author information is available at the end of the article
}

assemblers [7]. However, the choice of the $k$-mer size is crucial as too low or too high $k$-mer sizes lead to suboptimal assemblies. Indeed, low quality reads might produce false positive vertices, repeats lead to branching, while an uneven distribution of the reads results in gaps. The use of smaller $k$-mers reduces the problem associated with low quality reads and their uneven distribution, while larger $k$-mer sizes help to bridge repeat regions decreasing the branching problem [8]. In a balancing exercise, various $k$-mer sizes are usually selected, evaluating optimization by aiming for high N50 values and long, but fewer contigs. Whole Genome Mapping (WGM; Opgen Inc, Gaithersburg, MD, USA) is a relatively novel technique that generates high-resolution restriction maps of a genome based on the alignment of single DNA molecules cut with restriction enzymes and ordered with high resolution and accuracy [9]. WGM was proven helpful in mapping de novo assembled contigs against previously sequenced related genomes [10].

In this paper, we evaluated the utility of WGM for proper $k$-mer size selection and for optimization of parameters for de novo genome assembly. The whole genome sequence of a methicillin-resistant Staphylococcus 
aureus (MRSA) strain (E-MRSA15-CC22-SCCmecIV) was generated on an Illumina HiSeq-2000 via 2X150b paired end sequencing [11]. Reads were de novo assembled using Velvet, SPAdes and IDBA-UD employing a range of $k$-mers. Velvet, using a single $k$-mer approach ranging from $k$-mer size 81 to 123 showed an initial increase in N50 (until $k$-mer size 115) and longest contig size and a concomitant decrease in the total number of contigs with increasing $k$-mer size with both these parameters positively influencing the assembly outcome (Table 1). Ensuing, using WGM, a whole genome map of $S$. aureus EMRSA-15 was generated which, using MapSolver, was aligned with the assembly files corresponding to different $k$-mer sizes. Although the percentage of the genome covered by contigs increased with increasing $k$-mer size, a mis-assembly (spanning $119 \mathrm{~kb}$ ) was identified for the mapped contigs $(>40 \mathrm{~kb}$ ) for higher $k$-mer sizes (Table 1 ), revealing the best (without mis-assemblies) assembly was actually obtained using a $k$-mer size of 93 despite a higher N50 and fewer contigs as when for example utilizing a $k$-mer size of 115 (Table 1, Figure 1A, B). In contrast, SPAdes, which allows to combine a range of $k$-mer sizes in a multi-k-mer approach did not yield any mis-assemblies on this sequence for the N50 based best assemblies (Figure 1C). The same was true for IDBA, which similarly utilizes an iterative process including multiple $k$-mer sizes, while removing assembled sequences in subsequent rounds of analysis.

The general applicability of these results was investigated using two additional, similarly obtained, S. aureus sequences [UA-S391(accession \# CP007690) and Mu50CC5-SCCmecII (ATCC700699; previously sequenced and available under accession \# NC_002758], again revealing mis-assemblies for Velvet at the highest N50 values, while error free assemblies could be obtained for lower $k$-mer sizes (data not shown). In addition, the sequence of Klebsiella pneumoniae ST258 was similarly generated using Illumina HiSeq-2000 via 2X150b paired end sequencing and was assembled using all three assembly tools. In this case, apart from mis-assemblies seen for

Table 1 Assembly statistics of Velvet applied on Staphylococcus aureus (MRSA) strain E-MRSA15-CC22-SCCmecIV showing an increase in contig size and N50 when using higher $k$-mer sizes, but revealing a mis-assembly starting from k-mer size 97 using whole genome mapping

\begin{tabular}{|c|c|c|c|c|c|}
\hline K-mer size & N50 & Total number of contigs & Longest contig size & $\begin{array}{l}\text { Mis-assemblies } \\
\text { on mapped contigs* }\end{array}$ & $\begin{array}{l}\text { Approx. nts involved } \\
\text { in mis-assemblies }\end{array}$ \\
\hline \multicolumn{6}{|l|}{ Velvet } \\
\hline 81 & 162295 & 40 & 340060 & $1(10)$ & 122303 \\
\hline 83 & 170447 & 38 & 351373 & $1(9)$ & 122303 \\
\hline 85 & 170449 & 37 & 351321 & $0(10)$ & \\
\hline 87 & 173763 & 33 & 351326 & $0(10)$ & \\
\hline 89 & 173765 & 33 & 351394 & $0(10)$ & \\
\hline 91 & 173767 & 33 & 351330 & $0(10)$ & \\
\hline 93 & 173769 & 35 & 340092 & $0(10)$ & \\
\hline 97 & 175770 & 33 & 365247 & $1(9)$ & 130273 \\
\hline 99 & 175776 & 33 & 365260 & $1(10)$ & 130273 \\
\hline 101 & 187438 & 32 & 365623 & $1(9)$ & 130273 \\
\hline 103 & 187448 & 32 & 365625 & $1(9)$ & 130273 \\
\hline 105 & 187458 & 32 & 365638 & $1(9)$ & 130273 \\
\hline 107 & 187465 & 33 & 365647 & $1(9)$ & 130273 \\
\hline 109 & 212189 & 32 & 365656 & $1(9)$ & 130273 \\
\hline 111 & 212287 & 33 & 349286 & $2(8)$ & $93632 \& 153207$ \\
\hline 113 & 212292 & 34 & 349288 & $1(10)$ & 93634 \\
\hline 115 & 212294 & 34 & 349290 & $1(10)$ & 118928 \\
\hline 117 & 174074 & 35 & 349419 & $1(11)$ & 93634 \\
\hline 119 & 174076 & 35 & 349423 & $1(11)$ & 93638 \\
\hline 121 & 170642 & 37 & 349435 & $0(11)$ & \\
\hline 123 & 170654 & 38 & 340456 & $0(10)$ & \\
\hline
\end{tabular}

*Number of mapped contigs indicated between brackets. 


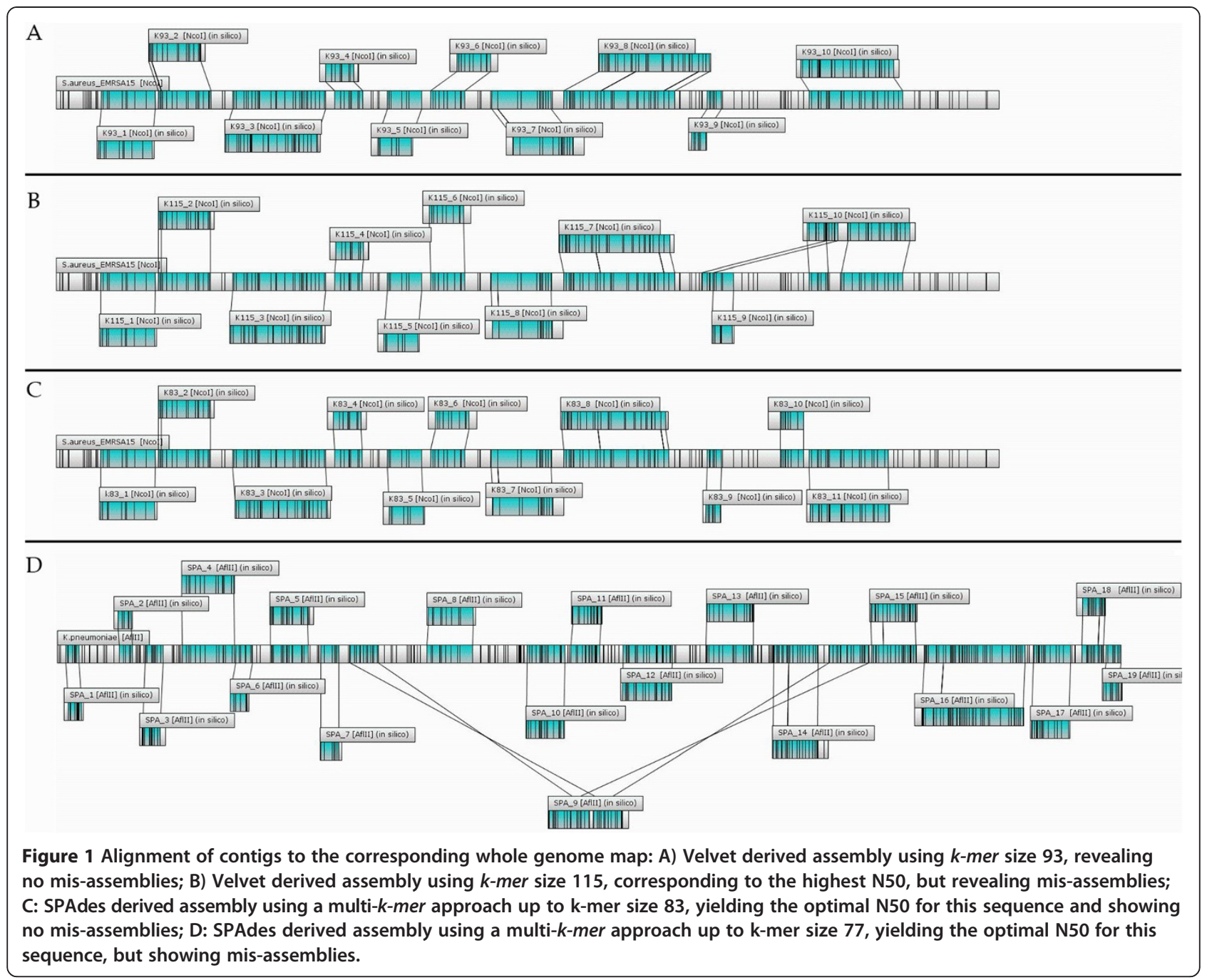

Velvet, also SPAdes and IDBA were shown to produce mis-assemblies for certain $k$-mer sizes (Figure 1D), further demonstrating the potential of WGM to identify misassemblies, even for assemblers utilizing multi-k-mer approaches (Additional file 1: Table S2).

\section{Conclusion}

Genome assembly based on de Bruijn graphs potentially yields mis-assemblies when only considering standard parameters such as total number and length of the contigs and N50. However, Whole Genome Mapping provides a powerful tool to identify such mis-assemblies and to select the optimal $k$-mer sizes to produce optimally assembled genomes. Despite of its additional cost, the biological need for error-free and complete genomes makes WGM an indispensable technique during the process of genome assembly and its validation.

\section{Additional file}

Additional file 1: Table S2. Assembly statistics of Velvet, SPAdes and IDBA-UD applied on Staphylococcus aureus (MRSA) strain E-MRSA15-CC22-SCCmedIV and assembly statistics of Velvet for Klebsiella pneumoniae showing an initial increase in contig size and N50 when using higher $k$-mer sizes, but revealing mis-assemblies associated with higher $\mathrm{k}$-mer sizes in some cases.

Competing interests

The authors declare that they have no competing interests.

\section{Authors' contributions}

BBX participated in the design of the study and performed the data analysis; JS participated in the design of the study and was involved in whole genome mapping; PM participated in conceiving and drafting the manuscript and performed the image processing; JPH and HdG provided the strains and were involved in sequencing for this study; HG and SMK participated in conceiving the study and revised the manuscript. All authors read and approved the final manuscript. 


\section{Acknowledgements}

Strains utilized in this study were obtained from the EU-FP6-MOSAR collection (MOZAR7-CC8-SCCmeclll; www.mosar-sic.org), the European Staphylococcal Typing Network, HARMONY (E-MRSA15-CC22-SCCmecIV) and from the Network on Antimicrobial Resistance in Staphylococcus aureus Program, NARSA (Mu50-CC5-SCCmecll).

\section{Funding}

This work is supported by funding from Research Foundation Flanders (FWO-F, Research project no. G.0983.10N), and the European Community (PREPARE network contract FP7-HEALTH F3 2013- no. 602525). B.B.X. is supported by University of Antwerp Research funds (BOF-DOCPRO 2012-27450).

\section{Author details}

Department of Medical Microbiology, Vaccine \& Infectious Disease Institute, Universiteit Antwerpen, Antwerp, Belgium. ${ }^{2}$ Viral Genetics Research Group, Vrije Universiteit Brussel, Brussels, Belgium. ${ }^{3}$ Structural Biology Brussels, Flanders Institute for Biotechnology (VIB), Vrije Universiteit Brussel, Brussels, Belgium.

\section{Received: 8 April 2014 Accepted: 21 July 2014}

Published: 30 July 2014

\section{References}

1. Li Y, Zheng H, Luo R, Wu H, Zhu H, Li R, Cao H, Wu B, Huang S, Shao H, Ma H, Zhang F, Feng S, Zhang W, Du H, Tian G, Li J, Zhang X, Li S, Bolund L, Kristiansen K, de Smith AJ, Blakemore AIF, Coin LJM, Yang H, Wang J, Wang J: Structural variation in two human genomes mapped at singlenucleotide resolution by whole genome de novo assembly. Nat Biotech 2011, 29:723-730.

2. Salzberg SL, Yorke JA: Beware of mis-assembled genomes. Bioinformatics 2005, 21:4320-4321.

3. Phillippy AM, Schatz MC, Pop M: Genome assembly forensics: finding the elusive mis-assembly. Genome Biol 2008, 9:R55.

4. Dark M: Whole-genome sequencing in bacteriology: state of the art Infect Drug Resist 2013, 6:115-123.

5. Zerbino DR, Birney E: Velvet: algorithms for de novo short read assembly using de Bruijn graphs. Genome Res 2008, 18:821-829.

6. Bankevich A, Nurk S, Antipov D, Gurevich AA, Dvorkin M, Kulikov AS, Lesin VM, Nikolenko SI, Pham S, Prjibelski AD, Pyshkin AV, Sirotkin AV, Vyahhi N, Tesler G, Alekseyev MA, Pevzner PA: SPAdes: a new genome assembly algorithm and its applications to single-cell sequencing. J Comput Bio/ 2012, 19:455-477.

7. Hunt M, Kikuchi T, Sanders M, Newbold C, Berriman M, Otto T: REAPR: a universal tool for genome assembly evaluation. Genome Biol 2013, 14:R47.

8. Peng Y, Leung HM, Yiu SM, Chin FL: IDBA - A Practical Iterative de Bruijn Graph De Novo Assembler. In Research in Computational Molecular Biology. Volume 6044. Edited by Berger B. Berlin Heidelberg: Springer; 2010:426-440. Lecture Notes in Computer Science].

9. Ananiev GE, Goldstein S, Runnheim R, Forrest DK, Zhou S, Potamousis K Churas CP, Bergendahl V, Thomson JA, Schwartz DC: Optical mapping discerns genome wide DNA methylation profiles. BMC Mol Biol 2008, 9:68.

10. Onmus-Leone F, Hang J, Clifford RJ, Yang Y, Riley MC, Kuschner RA, Waterman PE, Lesho EP: Enhanced De novo assembly of high throughput pyrosequencing data using whole genome mapping. PLoS One 2013, 8:e61762.

11. Sabirova JS, Xavier BB, Hernalsteens JP, De Greve H, leven M, Goossens H, Malhotra Kumar S: Complete genome sequences of Two prolific Biofilm-forming staphylococcus aureus isolates belonging to USA300 and EMRSA-15 clonal lineages. Genome Announc 2014, 2:e00610-14.

doi:10.1186/1756-0500-7-484

Cite this article as: Xavier et al:: Employing whole genome mapping for optimal de novo assembly of bacterial genomes. BMC Research Notes 2014 7:484

\section{Submit your next manuscript to BioMed Central and take full advantage of:}

- Convenient online submission

- Thorough peer review

- No space constraints or color figure charges

- Immediate publication on acceptance

- Inclusion in PubMed, CAS, Scopus and Google Scholar

- Research which is freely available for redistribution

Submit your manuscript at www.biomedcentral.com/submit 